\title{
LETTER FROM THE CO-EDITORS
}

$\mathrm{F}$ Tor the past four years, Christopher Pool and Gabriela Uruñuela y Ladrón de Guevara have valiantly served as Co-Editors of Latin American Antiquity (LAQ). Like all other journals, LAQ has sometimes sailed through turbulent waters. Thanks to Gabriela, Chris, and their remarkable Editorial Board, we begin our tenure at a time of calm. What once was an enormous backlog of articles has been reduced to a single pending issue. Moreover, because of a decision by the SAA Executive Board, what has been a severe problem-an acceptance rate that has fallen to just $20 \%$ because of page limitations - will be greatly eased. We are pleased to announce that each issue will increase by 24 pages. Put another way, we soon will be publishing within our four annual issues the page equivalent of a fifth issue. This will not only allow us to print more of the excellent articles that are submitted, but also should speed up time to publication.

In many ways, we hope to build upon what Chris, Gabriela, and their board have accomplished. One important focus of our tenure is to have more themed sections, that is, to publish groups of articles and reports on similar methodological or theoretical topics that describe research in different regions of Latin America and the Caribbean. We also will solicit short synthetic comments of the sort presented at the SAA meetings. This, we hope, will serve to create more cohesion and craft a journal that is more than a random collection of contributions.

A second goal, already underway, is to increase our presence in international indices. This will make publication in LAQ more valuable to scholars at institutions that use indices as metrics of scholarly impact. Third, through the work of our colleague Oswaldo Chinchilla, we hope to reinvigorate the Book Review section by publishing more reviews, review essays, and book notes covering the breadth of recent work in Latin American archaeology. Some of these will appear in the pages of LAQ, but many more may appear as online material. We extend a special invitation to authors and editors in Latin America to submit their recent books, which often go unnoticed outside their country of origin, for review.

We are pleased to have an excellent new Editorial Board that has already made important contributions. One of the critical functions of the board is that its members propose reviewers for all submissions. We hope this fosters even greater impartiality in the peer-review process.

The next few years will see changes in all the SAA publications. As is the case for many journals of the "hard sciences," more and more methodological statements, data, and supporting material will appear online as supplements rather than in the printed pages of LAQ itself. Printed articles will become shorter but we will print more of them. Finally, the spread of open access throughout the sciences and humanities will have important ramifications for our journal. We look forward to meeting these challenges and entering these exciting times together with our contributors and readers.

We both will attend the Conferencia Intercontinental to be held in Lima, Perú, August 8-10, 2014. We look forward to talking with you about the future direction of LAQ and welcome your suggestions on how to make the journal more inclusive and of greater value to all archaeologists working in Latin America and the Caribbean.

Geoffrey E. Braswell and María A. Gutiérrez

Latin American Antiquity 25(2), 2014, pp. 123-124

Copyright $(2014$ by the Society for American Archaeology 


\section{CARTA DE LOS CO-EDITORES}

$\mathrm{D}$ urante los últimos cuatro años, Christopher Pool y Gabriela Uruñuela y Ladrón de Guevara han servido de forma muy valiente como co-editores de Latin American Antiquity. Al igual que todas las demás revistas, LAQ ha navegado algunas veces a través de aguas turbulentas. Sin embargo, gracias a Gabriela, Chris y su notable Comité Editorial, comenzamos nuestro periodo editorial en un momento de calma. Ellos consiguieron reducir lo que una vez fue una enorme acumulación de artículos en un único número pendiente. Por otra parte, y gracias a una decisión del Comité Ejecutivo de la SAA, lo que ha sido un grave problema hasta el momento -una tasa de aceptación de trabajos del $20 \%$ debido a las limitaciones de páginas- se verá muy aliviado con dicha decisión. Nos complace anunciar que cada nuevo número se incrementará en 24 páginas, es decir, un total de 128 páginas anuales. Esto no sólo nos permitirá ampliar el número de excelentes artículos que podremos publicar, sino también nos permitirá acelerar el tiempo de su publicación.

En muchos aspectos, esperamos seguir construyendo sobre lo que Chris, Gabriela y su Comité Editorial ya han logrado. Un objetivo importante de nuestro mandato es tener más secciones temáticas, es decir, publicar grupos de artículos sobre temas metodológicos y/o teóricos similares que describen la investigación en diferentes regiones de América Latina y el Caribe. También vamos a solicitar comentarios sintéticos cortos, del tipo presentado en las reuniones de la SAA. Esto, esperamos, servirá para crear una mayor cohesión y como resultado, obtener una revista que es mucho más que una colección de artículos al azar.

Un segundo objetivo, ya en marcha, es aumentar nuestra presencia en los índices internacionales. Creemos que esto redundará en una mayor valoración de las publicaciones en LAQ para los colegas de instituciones que utilizan los índices como indicadores de impacto académico. En tercer lugar, a través del trabajo de nuestro colega Oswaldo Chinchilla, esperamos poder revitalizar la sección Book Review publicando más revisiones, ensayos críticos y notas de libros que cubren la amplia diversidad de trabajos recientes de la arqueología latinoamericana. Algunas de ellas aparecerá en las páginas de LAQ, pero muchas más pueden publicarse como material on line. Extendemos una invitación especial a los autores y editores de América Latina a presentar para su revisión sus últimos libros, los cuales a menudo pasan desapercibidos fuera de su país de origen.

Estamos muy contentos de tener un excelente nuevo Comité Editorial que ya ha hecho importantes contribuciones. Una de las funciones críticas de este comité es aportar y discutir junto con los coeditores los nombres y pertenencia de los diferentes revisores que evalúan los trabajos enviados. Esperamos que esta forma de trabajar fomente aún más la imparcialidad en el proceso de revisión por pares.

En los próximos años van a ocurrir cambios en todas las publicaciones de la SAA. Tal como sucede en muchas revistas de las "ciencias duras", más y más desarrollos metodológicos, datos y material de apoyo van a aparecer on line en forma de suplementos en lugar de hacerlo en las páginas impresas de LAQ. Los artículos impresos serán más cortos, aunque imprimiremos mayor número de ellos. Por último, la gran difusión del concepto de acceso abierto a través de las ciencias y las humanidades tendrá diversificaciones importantes para nuestra revista. Esperamos poder responder a estos desafíos y recorrer estos tiempos excitantes de cambios acompañados por nuestros colaboradores $y$ lectores.

Nosotros, como co-editores de LAQ, estaremos presentes en la próxima Conferencia Intercontinental, que se celebrará en Lima, Perú, entre el 8 y 10 de agosto de 2014. Nos gustaría poder encontrarnos allí y tener la oportunidad de hablar con más detalles de nuestros objetivos y direcciones futuras de LAQ. Además, te invitamos a discutir con nosotros lo que usted cree que podría ayudar a hacer más inclusiva a la revista y de mayor interés para todos los que trabajan en arqueología latinoamericana y caribeña.

María A. Gutiérrez y Geoffrey E. Braswell 